\title{
Semi-Automated DigitalMicrograph Routine for Real-Time Phase Identification
}

\author{
Edward R. White ${ }^{1}$, Jonathan Weiner ${ }^{1}$, Andrés García-Trenco ${ }^{1}$, Sebastian D. Pike ${ }^{1}$, Charlotte K. \\ Williams ${ }^{1}$, and Milo S. P. Shaffer ${ }^{1}$ \\ 1. Department of Chemistry, Imperial College London, South Kensington, London, SW7 2AZ, UK.
}

Individual nanocrystallite composition can be determined by comparing high resolution transmission electron microscopy (HRTEM) measurements of lattice spacings to the known lattice spacings of materials [1]. Without the aid of expensive software, however, this process can be overwhelmingly time consuming for even a single image containing multiple crystals [2]. Here, we describe a semi-automated routine that enables the user to rapidly identify the phases present in HRTEM images using the native acquisition software on most commercially available TEMs.

The algorithm, described below, has two main functions. The first is to overlay rings corresponding to some or all known lattice spacings of a chosen material in an electron diffractogram. The second is to filter the electron diffractogram at the selected spacings and then show in real space where each phase is located. The routine produces an intuitive graphical user interface (GUI) directly in DigitalMicrograph, which allows image processing without data type conversion or third-party software processing [3].

When first running the routine, the user inputs a text file containing the lattice spacings and corresponding hkl values for each possible phase present. The routine parses output text files from a standard X-ray diffraction database; adaptation to other formats is straightforward. As shown in Figure 1, the program loads a GUI containing lists of several different phases and their corresponding hkl values. The example shown in Figure 2 is a post-catalysis mixture of zinc and copper nanoparticles, where the exact composition is unknown. Here, we have chosen to examine the possible presence of zinc oxide, metallic copper, copper oxides, copper hydride, brass, metallic zinc, and zinc stearate.

Pressing 'Generate FFT' in the GUI computes the fast-Fourier-transform of the HRTEM image and displays the resulting electron diffractogram. Selecting a material in the GUI then overlays rings on the electron diffractogram at each material's known lattice spacings. As shown in Figure 2c, rings at individual spacings are displayed by selecting only those corresponding hkl values. In this manner, determining which phases are present is done rapidly by inspection; in this case both zinc oxide and metallic copper are identified.

Once the user identifies all present phases, the contribution of each to the original HRTEM image is highlighted. Selecting 'IFFT on selected' in the GUI places ring-shaped masks of adjustable width and Gaussian tail length on the electron diffractogram at each selected spacing, generating an additional electron diffractogram for each masked region. The inverse-fast-Fourier-transform is then computed for each new electron diffractogram. As shown in Figure $2 \mathrm{~d}-\mathrm{e}$, this routine produces real space images localizing each phase. The contribution from each hkl selection can be displayed separately to indicate specific lattice orientations if desired (not shown). Finally, the resultant real space images are colored and overlaid on the original HRTEM image. Figure $2 \mathrm{f}$ shows such an image, in this case the HRTEM image contains a cluster of $\mathrm{ZnO}$ nanoparticles surrounding a metallic $\mathrm{Cu}$ nanoparticle. The ability to quickly identify regions containing an interface between catalyst species should lead to a better understanding of the active reaction sites in nanoparticle catalytic systems [4]. 
References:

[1] DB Williams and CB Carter in "Transmission Electron Microscopy" (Springer, New York) p. 283.

[2] EF Rauch and M Véron, Materials Characterization 98 (2014) p. 1.

[3] DRG Mitchell, Microscopy Research and Technique 71 (2008) p. 588.

[4] The authors acknowledge funding from EPSRC grant number EP/K035274/1. Dr. Bernhard Schaffer is thanked for his advice with scripting DigitalMicrograph.

\begin{tabular}{|c|c|c|c|}
\hline \multicolumn{3}{|c|}{$F=$ Compare HRTEM and XRD data } & 0 回 $-x$ \\
\hline Image operations & -materials - & -hkl's- & {$\left[\begin{array}{lll}R & G & B\end{array}\right]$} \\
\hline & $\ulcorner\mathrm{Cu}$ & $\nabla 111 \Gamma 200 \Gamma 220 \Gamma 311 \Gamma 222 \Gamma 400 \Gamma 331 \Gamma 420$ & $\nabla\ulcorner\ulcorner$ \\
\hline Crop ROI & $\ulcorner\mathrm{ZnO}$ & $\nabla 100 \mathrm{~V} 002 \mathrm{~V} 101 \mathrm{~V} 102 \mathrm{~V} 110 \mathrm{~V} 103 \Gamma 200 \mathrm{~V} 112 \Gamma 201 \Gamma 004 \mathrm{~V} 202 \Gamma 104 \mathrm{~V} 203\ulcorner 210\ulcorner 211$ & $\ulcorner\nabla\ulcorner$ \\
\hline Generate FFT & \ulcorner & $\Gamma 114 \Gamma 212 \Gamma 105 \Gamma 204 \Gamma 300\ulcorner 213 \Gamma 302 \Gamma 006 \Gamma 205 \Gamma 106 \Gamma 214 \Gamma 220$ & 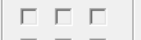 \\
\hline & $\ulcorner\mathrm{Cu20}$ & $\ulcorner 110 \Gamma 111 \Gamma 200 \Gamma 211 \Gamma 220 \Gamma 310 \Gamma 311 \Gamma 222 \Gamma 400 \Gamma 331 \Gamma 420 \Gamma 422 \Gamma 511$ & $г г$ \\
\hline$\ulcorner$ FFT operations & $\ulcorner$ CuO_1 & $\Gamma-110\ulcorner 002 \Gamma 111 \Gamma 200 \Gamma-112 \Gamma-202 \Gamma 112 \Gamma 020\ulcorner 202 \Gamma-113 \Gamma 022 \Gamma-311 \Gamma 113 \Gamma-220 \Gamma 311$ & 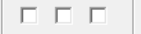 \\
\hline Adjust & & $\Gamma 004 \Gamma-222 \Gamma-204 \Gamma-313 \Gamma 222 \Gamma 400 \Gamma-402 \Gamma-131 \Gamma 131 \Gamma 204 \Gamma 024 \Gamma 313 \Gamma-115 \Gamma-224 \Gamma-420$ & $\ulcorner\ulcorner\ulcorner$ \\
\hline calibration & & $\ulcorner-133\ulcorner-422 \Gamma-404 \Gamma 115 \Gamma-331 \Gamma 133 \Gamma-511$ & $\ulcorner\ulcorner\ulcorner$ \\
\hline$[1.0 \Delta \simeq$ & $\left\ulcorner\mathrm{CuO} \_2\right.$ & $\ulcorner 110\ulcorner 002 \Gamma 11-1 \Gamma 111 \Gamma 200\ulcorner 11-2 \Gamma 20-2 \Gamma 112 \Gamma 020\ulcorner 021 \Gamma 202 \Gamma 11-3 \Gamma 022 \Gamma 31-1 \Gamma 310$ & $\ulcorner\ulcorner\ulcorner$ \\
\hline & & $\ulcorner 113 \Gamma 220 \Gamma 22-1 \Gamma 31-2 \Gamma 311 \Gamma 221 \Gamma 004 \Gamma 22-2 \Gamma 023 \Gamma 20-4 \Gamma 11-4 \Gamma 31-3 \Gamma 222 \Gamma 312 \Gamma 40-2$ & $\ulcorner\ulcorner г$ \\
\hline - Inverse + St- & $\Gamma$ & $\ulcorner 22 \cdot 3\ulcorner 114 \Gamma 130 \Gamma 13-1 \Gamma 131 \Gamma 204 \Gamma 223 \Gamma 313\ulcorner 402 \Gamma 11-5 \Gamma 22-4 \Gamma 420 \Gamma 13-3 \Gamma 42-2 \Gamma 40-4$ & $\ulcorner\ulcorner\ulcorner$ \\
\hline - Stack & $\Gamma$ & $\ulcorner 115\ulcorner 421 \Gamma 133 \Gamma 51-1 \Gamma 224 \Gamma 331$ & $\ulcorner\ulcorner\ulcorner$ \\
\hline C Separte & $\ulcorner\mathrm{CuH}$ & $\ulcorner 100\ulcorner 002 \Gamma 101 \Gamma 102 \Gamma 110 \Gamma 103 \Gamma 112$ & $\ulcorner\ulcorner\ulcorner$ \\
\hline$\sqrt{V}$ Colonized & $\Gamma$ Cu_64ZZ_36 & $\ulcorner 111\ulcorner 200\ulcorner 220\ulcorner 311 \Gamma 222 \Gamma 400\ulcorner 331 \Gamma 420$ & $\ulcorner\ulcorner\ulcorner$ \\
\hline Use abs val & $\ulcorner\mathrm{Zn}$ & $\ulcorner 002\ulcorner 100\ulcorner 101\ulcorner 102\ulcorner 103\ulcorner 110\ulcorner 004 \Gamma 112 \Gamma 200\ulcorner 201 \Gamma 104 \Gamma 202 \Gamma 203\ulcorner 105 \Gamma 114$ & 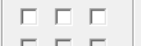 \\
\hline 12 Ring width & & $\ulcorner 210\ulcorner 211\ulcorner 204 \Gamma 006 \Gamma 212$ & $\ulcorner\ulcorner\ulcorner$ \\
\hline 5 Gaussian tail & $\ulcorner$ Zn stearate & 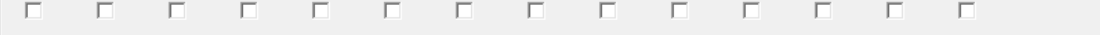 & ггг \\
\hline $\begin{array}{l}3 \text { Sigma multiplier } \\
\text { IFFT on selected }\end{array}$ & & & \\
\hline & & edv & \\
\hline
\end{tabular}

Figure 1. Graphical user interface for real-time phase identification DigitalMicrograph routine.
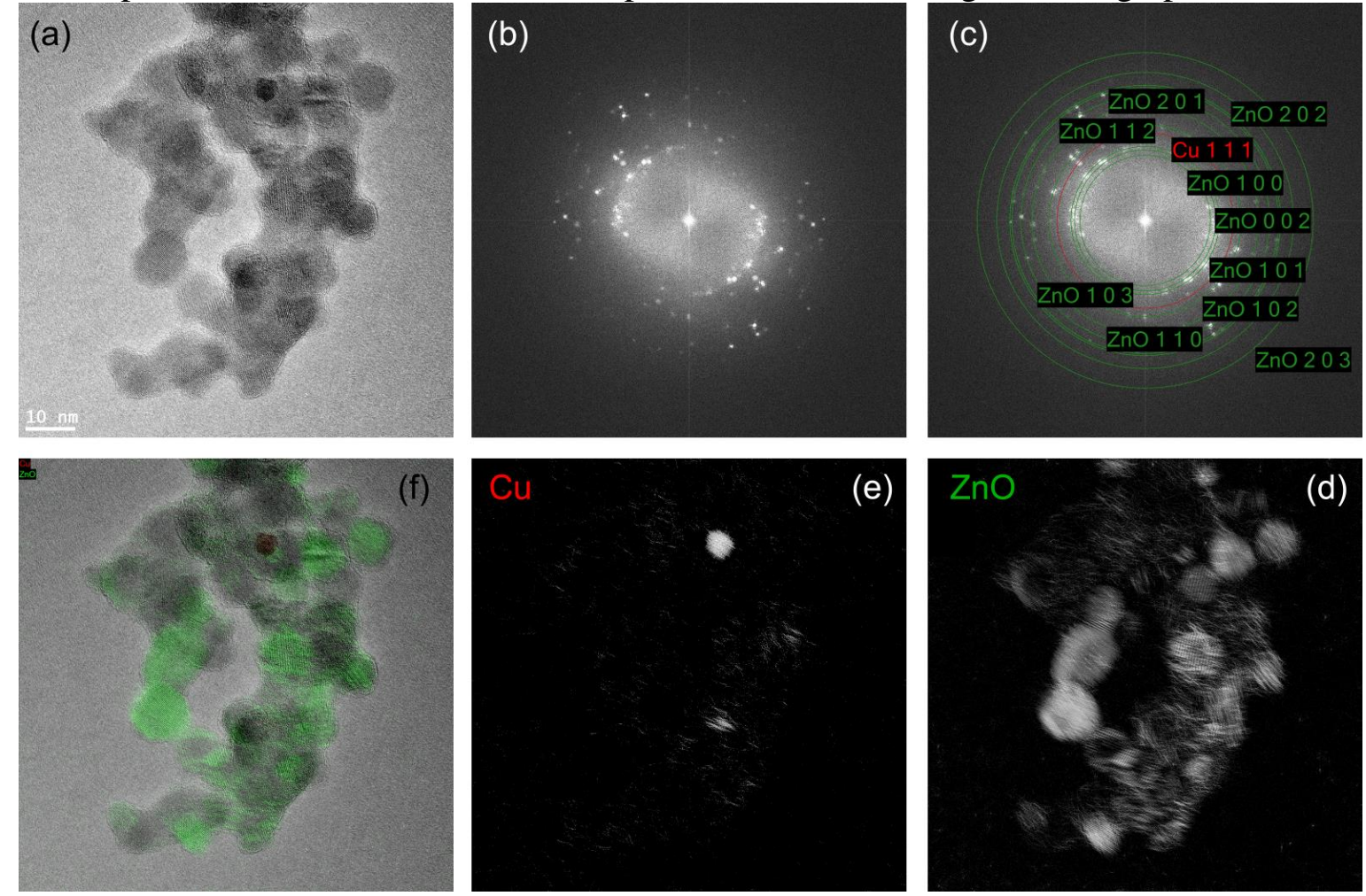

Figure 2. Example of routine in operation. (a) HRTEM image of nanoparticle cluster. (b) Electron diffractogram computed from (a). (c) Phase identification by selecting the hkl values shown in Figure 1. (d) Sum of all IFFTs with corresponding filters placed at $\mathrm{ZnO}$ spacings. (e) IFFT with a filter placed at the $\mathrm{Cu}(111)$ spacing. (f) Overlay of images (d) and (e) colored green and red respectively, on (a). 